\title{
Shape Stability of Polyethylene Glycol/Acetylene Black Phase Change Composites for Latent Heat Storage
}

\author{
Jingjing Zhang $\mathbb{D}^{1},{ }^{1}$ Hairong Li $\mathbb{C}^{2},{ }^{2}$ Junyang Tu $\mathbb{D}^{1},{ }^{1}$ Ruonan Shi $\mathbb{D}^{1},{ }^{1}$ Zhiping Luo $\mathbb{D}^{1,3}$ \\ Chuanxi Xiong, ${ }^{1}$ and Ming Jiang $\mathbb{D}^{1}$ \\ ${ }^{1}$ School of Materials Science and Engineering, State Key Lab for New Textile Materials and Advanced Processing Technology, \\ Wuhan Textile University, Wuhan 430200, China \\ ${ }^{2}$ Mechanical Metrology Division, Hubei Institute of Measurement and Testing Technology, Wuhan 430223, China \\ ${ }^{3}$ Department of Chemistry and Physics, Fayetteville State University, Fayetteville, NC 28301, USA
}

Correspondence should be addressed to Hairong Li; ronghailee@163.com and Ming Jiang; mjiang@wtu.edu.cn

Received 1 August 2018; Accepted 1 October 2018; Published 24 October 2018

Academic Editor: Veronica Calado

Copyright ( 2018 Jingjing Zhang et al. This is an open access article distributed under the Creative Commons Attribution License, which permits unrestricted use, distribution, and reproduction in any medium, provided the original work is properly cited.

\begin{abstract}
Sufficient shape stability is essential for a high-performance phase change material (PCM). Although significant advances have been made to develop form-stable composites, technical development in the field of polymer-based PCMs is currently limited by an incomplete understanding of the shape stability. Form-stable polyethylene glycol/acetylene black (PEG/AB) PCMs containing PEGs with different average molecular weights have been obtained by melt mixing to investigate the shape stability of the PEG/AB composites. It was found that the phase change behaviors of the PEG/AB composites were not only attributed to the interactions between the $\mathrm{AB}$ and PEG, but also to the intermolecular interactions of the PEG chains, depending on the varying molecular weights of the PEGs. Physically crosslinked structure with temporary junctions was formed through hydrogen bonding, capillary, surface tension forces, intermolecular friction, and macromolecular entanglement, which contributed to the constrained chain motion and thus the solid-solid phase change behavior of the PEG/AB composites. The physically crosslinked structure was more stable with longer length of the PEG molecular chains, resulting in higher critical impregnated contents of the PEG into the AB and thus improved latent heat.
\end{abstract}

\section{Introduction}

Energy storage can optimize the energy flows to overcome the mismatch between the demand for energy and the supply of energy, especially for energy generated by variable and renewable resources, such as water, wind, and solar energy [1]. Among the principal energy-storage technologies, phase change materials (PCMs) have drawn tremendous attention because they can store and release large amounts of latent heat as they change from one physical state to another for effective use of thermal energy [2-5]. To increase the energystorage ability, PCMs with large latent heat are essential for future thermal energy storage and management $[6,7]$.

Among the PCMs investigated, polyethylene glycol (PEG) is one of the prospective candidates in this regard as it exhibits relatively high latent heat-storage capacity, tunable solid-liquid phase change temperature, and noncorrosiveness, which leads to compact storage devices with high energy-storage density. Moreover, PEG has much better cycling stability during services and higher security than small-molecule organic PCMs due to nondetectable vapor pressure when melts [8-11]. Nevertheless, as a solidliquid PCM, the applications of PEG are largely limited, as the liquid phase leaks above the phase change point. The leakage is usually circumvented by introducing form-stable support. Considerable efforts have been devoted to develop form-stable composites with high energy-storage densities [12-23]. The form stabilization strategies of PCMs include encapsulation [12, 13], grafting [14, 15], and physical adsorptions [16-23]. Clay, diatomite, halloysite, active carbon, silica, expanded graphite, and graphene have been employed as fillers to prevent melted PCMs from leaking during the 
phase transition via physical adsorptions, relying on their predominantly polar surface and/or high porous structures [16-23]. However, although significant advances have been made, technical development in the field of polymer-based PCMs is currently limited by an incomplete understanding of the shape stability. The affinity of support fillers to PCMs depends on their structures, so the structure of the fillers usually is a primary consideration for the shape stability. Thus far, few attentions have been paid on the difference in shape stabilities between polymeric PCMs and low molecular weight organic PCMs. The effect of some critical structure factors for polymeric PCMs, such as molecular weight, on the shape stability was largely neglected. However, the shape stability of polymer-based composite PCMs could not be entirely determined by the affinity of support fillers to PCMs or the interactions between them and might be partly attributed to the structure of the polymeric PCMs. Therefore, a further understanding of the form stabilization mechanisms would ultimately produce a more rational and general approach to the thermal property optimisation of PEG-based composite PCMs.

The present work is an attempt to develop a better understanding of the shape stability of polymeric PCMbased composites by comparing the effects of PEGs with different molecular weights $\left(M_{\mathrm{w}}\right)$ on the thermal properties of PCM composites filled by commercially available acetylene black $(\mathrm{AB})$. The phase change behaviors of the formstable PEG/AB PCMs were verified by polarized optical microscopy (POM) and filter paper test. Furthermore, the structures and thermophysical properties of the composite PCMs were analyzed by scanning electron microscopy (SEM), X-ray diffractometer (XRD), thermogravimetric analysis (TGA), and differential scanning calorimetry (DSC). Finally, the existence of an intermolecular interaction mechanism of solid-solid phase change besides simple physical adsorptions in the PEG/AB composite PCMs was found.

\section{Materials and Methods}

2.1. Materials. Chemically pure PEGs were purchased from Sinopharm Chemical Reagent Beijing Co., Ltd. PEG800, PEG4000, PEG6000, and PEG10000 were utilized in this work. AB (purity $>99.99 \%$, particle size $=150-200 \mathrm{~nm}$ based on the supplier's information) was purchased from Shanghai Chemical Reagent Co. Ltd. and used after drying.

2.2. Preparation of PEG/AB Composites. To fabricate the PEG/AB composites, the PEGs with various average molecular weights were first melt at $50-70^{\circ} \mathrm{C}$, and then the $\mathrm{AB}$ was slowly added under stirring and vacuum. Finally, the mixture was dried in a vacuum oven at $70^{\circ} \mathrm{C}$ for $48 \mathrm{~h}$.

2.3. Characterization. The Fourier-transform infrared (FTIR) spectrum was observed at room temperature (Thermo Nicolet Nexus) in the range of 4,000 to $400 \mathrm{~cm}^{-1}$ using $\mathrm{KBr}$ pellets. Nitrogen adsorption/desorption isotherms were measured on a Micromeritics Model TriStar II 3020 volumetric analyzer (USA). POM observation was conducted on an Olympus BX51-P polarizing optical microscope equipped with a LTS 350 hot stage. TGA was carried out on a TG/DTA 220U (Seiko Instrument Co. Ltd, Tokyo Japan) with the Exstar 6000 Station. The samples were scanned from 20 to $800^{\circ} \mathrm{C}$ with the heating rate of $10^{\circ} \mathrm{C} / \mathrm{min}$ and nitrogen gas purging. The $\mathrm{X}$-ray measurements were carried out on a Rigaku D/Max-IIIA $\mathrm{X}$-ray diffractometer with $\mathrm{Cu}-\mathrm{Ka}$ radiation at a wavelength of $1.54 \AA$. Thermal analysis was performed using a DSC (PE Co., USA). The thermal conductivities of the various samples were measured at $28^{\circ} \mathrm{C}$ by the guarded heat flow test method (TA Instruments DTC 300, USA). Thermograms were obtained at a heating and cooling rate of $5^{\circ} \mathrm{C} \cdot \mathrm{min}^{-1}$ at a temperature range from $20^{\circ} \mathrm{C}$ to $80^{\circ} \mathrm{C}$ under nitrogen atmosphere. To confirm the reproducibility of the results, at least two samples were measured for each composition. For each sample, at least three DSC traces were recorded. SEM measurements were performed with JSM-IT300A (JEOL Ltd., Japan).

\section{Results and Discussion}

3.1. Chemical and Physical Characterizations of the $A B$ Particles. Chemical characterizations of the $\mathrm{AB}$ particles and the PEG/AB composites are carried out using FTIR spectroscopy, as shown in Figure 1. In the spectrum of the $A B$ (Figure 1(a)), the peaks at $2,888 \mathrm{~cm}^{-1}, 1,146 \mathrm{~cm}^{-1}$, and $1,114 \mathrm{~cm}^{-1}$ represent the stretching vibration of $\mathrm{C}-\mathrm{O}$ groups. The peak at $1,637 \mathrm{~cm}^{-1}$ is attributed to $\mathrm{C}=\mathrm{O}$ stretching vibration. $\mathrm{O}-\mathrm{H}$ stretching vibrations are characterized by the broadband in the region of $3,300-3,650 \mathrm{~cm}^{-1}$. The spectrum of pristine PEG (Figure $1(\mathrm{~b})$ ) is characterized by $\mathrm{O}-\mathrm{H}$ stretching vibration at $3433 \mathrm{~cm}^{-1}$ and the strong absorption peaks of $\mathrm{C}-\mathrm{H}$ bonds at $2888 \mathrm{~cm}^{-1}, 953 \mathrm{~cm}^{-1}$, and $839 \mathrm{~cm}^{-1}$. The $\mathrm{C}-\mathrm{O}$ stretching vibration was at $1106 \mathrm{~cm}^{-1}$. The FTIR results indicate that no significant new peaks were observed other than characteristic peaks of PEGs and AB at FTIR spectrums of the PEG/AB composites (Figure 1(b)), which proves that the interactions between the functional groups of PEG and $A B$ are physical in nature. Moreover, the existence of weak hydrogen bonding between the $A B$ and PEG, which could be able to increase PEG-absorbing capacity of the AB to some extent.

To further examine the pore adsorption capacity of the $\mathrm{AB}$ particles, nitrogen adsorption/desorption isotherms are measured. The pore-size distribution of the $A B$ is shown in Figure 2. The $A B$ particles possess a specific Brunauer-Emmett-Teller (BET) surface area of $53.79 \mathrm{~m}^{2} / \mathrm{g}$, with a most probable pore size of about $2.6 \mathrm{~nm}$. The specific surface area is much lower than those of porous carbon materials, such as expanded graphite or active carbon. Moreover, the average pore size of the AB particles is smaller than the equivalent sphere diameter of PEG4000 or PEG with higher $M_{\mathrm{w}}$. The equivalent radius of $P E G$ random coils can be calculated according to previous reports [24]. The above analysis shows a relatively low pore adsorption capacity of the $A B$ particles. 


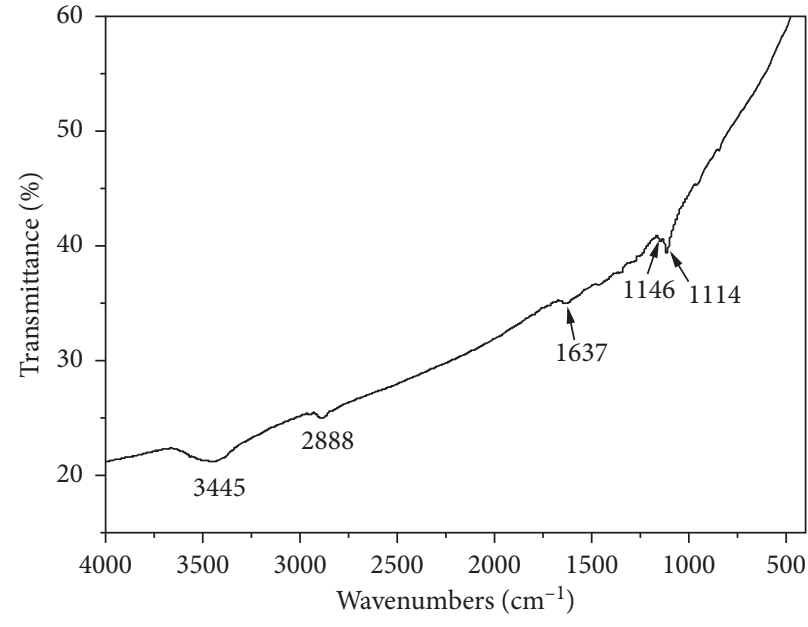

(a)

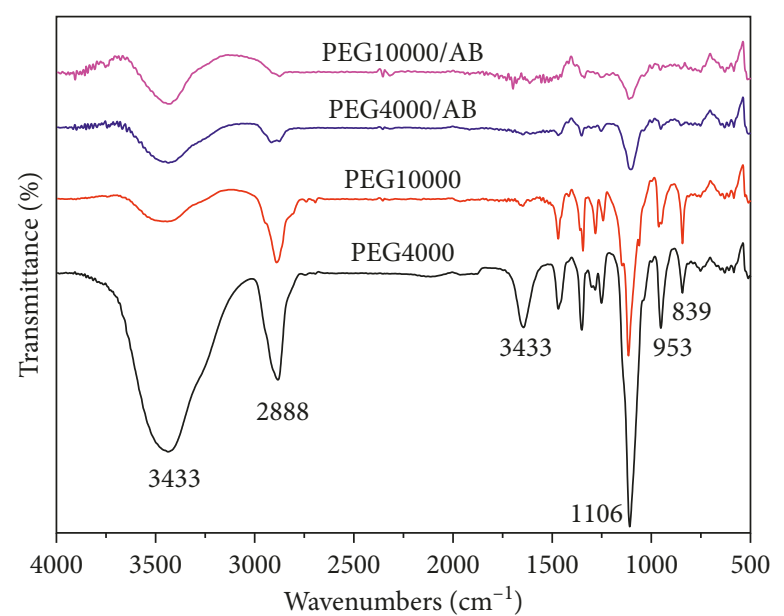

(b)

Figure 1: FTIR spectrums of the AB particles (a) and PEG/AB composites (b).

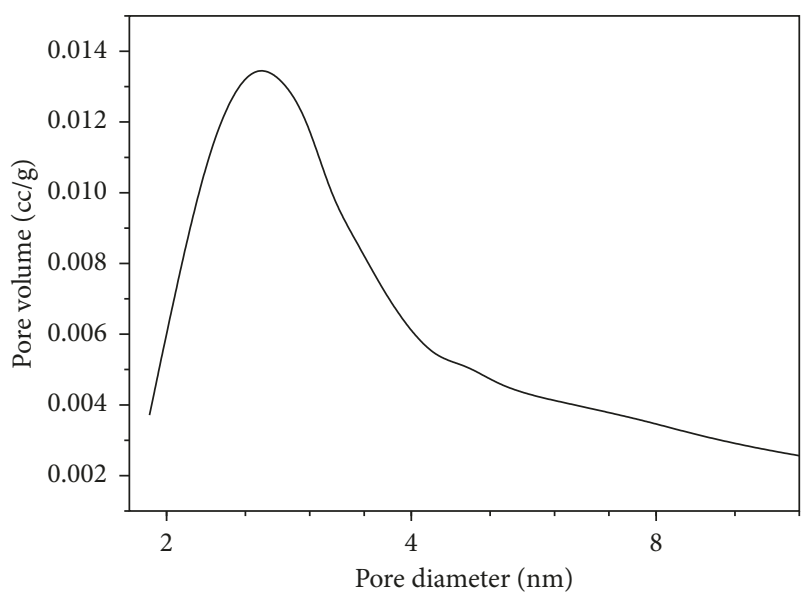

FIgURe 2: Pore-size distribution of the $\mathrm{AB}$ particles.

3.2. Leakage Behavior of the PEG/AB Composites. The leakage behavior of the PEG/AB composites is investigated by POM and filter paper test. The POM images are taken below the crystallization temperature of the PEG10,000/AB composites, as shown in Figure 3. The darker regions correspond to the saturated $A B$, whereas the lighter regions marked by arrows indicate the excess PEG, as shown in Figure 3(a). When the mass fraction of the $\mathrm{AB}$ is increased to $11.6 \mathrm{wt} . \%$, the excess PEG1,000 cannot be observed in the POM image in Figure 3(b), indicating the complete absorption of the $\mathrm{PEG}$ in the $\mathrm{AB}$ particles.

The leakage behavior of the PEG/AB composites at elevated temperature above the phase change point is further studied. Typically, the PEG/AB samples are kept in filter paper envelopes above the phase change temperatures for $1.5 \mathrm{~h}$ and the change in the weight of the filter paper envelopes before and after the test are measured, and the results were presented in Table 1. No significant change in weight is observed under $80^{\circ} \mathrm{C}$, indicating that no obvious PEG leakage occurred during the test. Consequently, the optimum absorption ratio of
PEG800, PEG4,000, PEG6,000, and PEG10,000 in AB should be around $0.76,0.84,0.87$, and 0.88 (responding to the results obtained by TGA below), respectively, and these mass fractions are adequate to obtain form-stable PEG/AB composite PCMs.

3.3. Thermal Reliability of the PEG/AB Composites. The TG curves of the pure PEG and PEG/AB composite PCMs are shown in Figure 4. As can be seen from the curves, all samples have a single thermal degradation stage. The degradation of the PEGs with relatively large molecular weights and their composite PCMs starts around $350^{\circ} \mathrm{C}$ and ends at $430^{\circ} \mathrm{C}$, while the PEG800 starts below $300^{\circ} \mathrm{C}$ and ends at $430^{\circ} \mathrm{C}$. It indicates that the $\mathrm{AB}$ does not significantly affect the thermal stability of the PEGs with relatively large $M_{\mathrm{w}}$ but improves the thermal stability of PEG with relatively small $M_{\mathrm{w}}$ (PEG800). This is attributed to the stronger interaction between AB and PEG800 induced by pore adsorption due to the smaller equivalent sphere diameter of PEG800 than the average pore size of $\mathrm{AB}$. That is, the PEG chains are trapped inside the $A B$ pores. In addition, these results also demonstrate that no chemical reactions take place during simple melt blending and impregnation process. Moreover, the TG curves of the form-stable composite PCMs also confirm the optimum impregnation ratios of PEG800, PEG4,000, PEG6,000, and PEG10,000 as 76.4 wt\%, 83.9 wt\%, 86.8 wt $\%$, and $88.4 \mathrm{wt} \%$, respectively.

3.4. Crystalline Properties of the PEG/AB Composites. The $\mathrm{XRD}$ patterns of the PEG and PEG/AB composites at room temperature are displayed in Figure 5. It is observed that the $\mathrm{AB}$ only has a weak and broad peak at $25^{\circ}$, indicating an amorphous structure. The XRD patterns of the PEG4,000, PEG6,000, and PEG10,000 are characterized by two strong $2 \theta$ peaks at $19.1^{\circ}$ and $23.6^{\circ}$, which are assigned to the typical plane of (120) and (112) of the PEGs. The XRD curves of the PEG/AB composites are similar to those pure PEGs, and the position of the diffraction peaks remains unchanged after the 


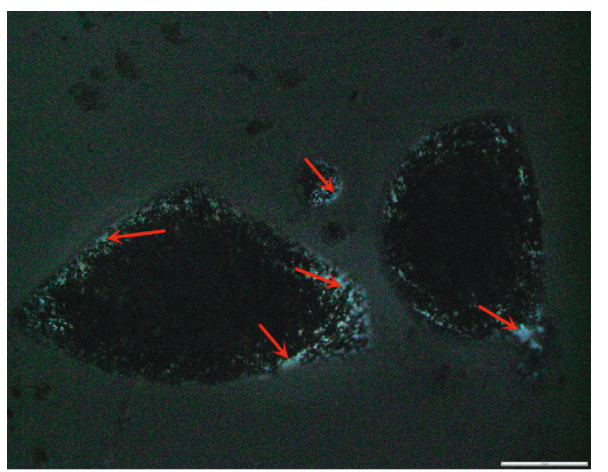

(a)

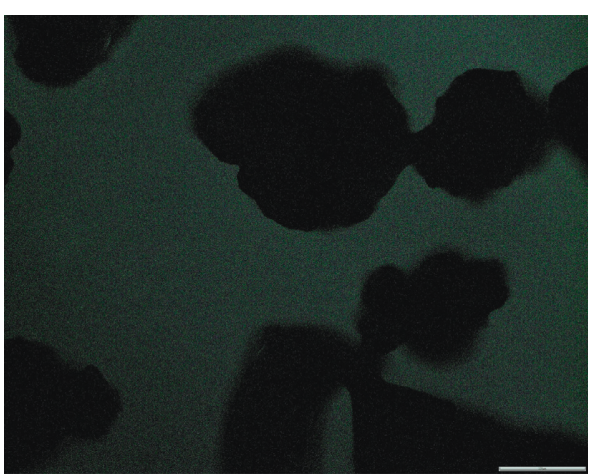

(b)

FIGUre 3: POM images taken below the crystallization temperature of PEG10000/9 wt.\%AB (a) and PEG10000/11.6 wt.\%AB (b) Scale bar: $100 \mu \mathrm{m}$.

TABLe 1: Relative weight changes of the PEG/AB composites before and after the leakage tests at $80^{\circ} \mathrm{C}$ for $1.5 \mathrm{~h}$.

\begin{tabular}{lcccc}
\hline Samples & Average weight before $(\mathrm{mg})$ & Average weight ${ }_{\text {after }}(\mathrm{mg})$ & $\Delta(\%)$ & Standard deviation $(\mathrm{mg})$ \\
\hline PEG800/AB & 313.2 & 317.2 & 1.27 & 0.88 \\
PEG4000/AB & 318.5 & 321.2 & 0.85 & 0.31 \\
PEG6000/AB & 319.4 & 322.7 & 1.03 & 0.76 \\
PEG10000/AB & 312.3 & 315.3 & 0.96 & 0.56 \\
\hline
\end{tabular}

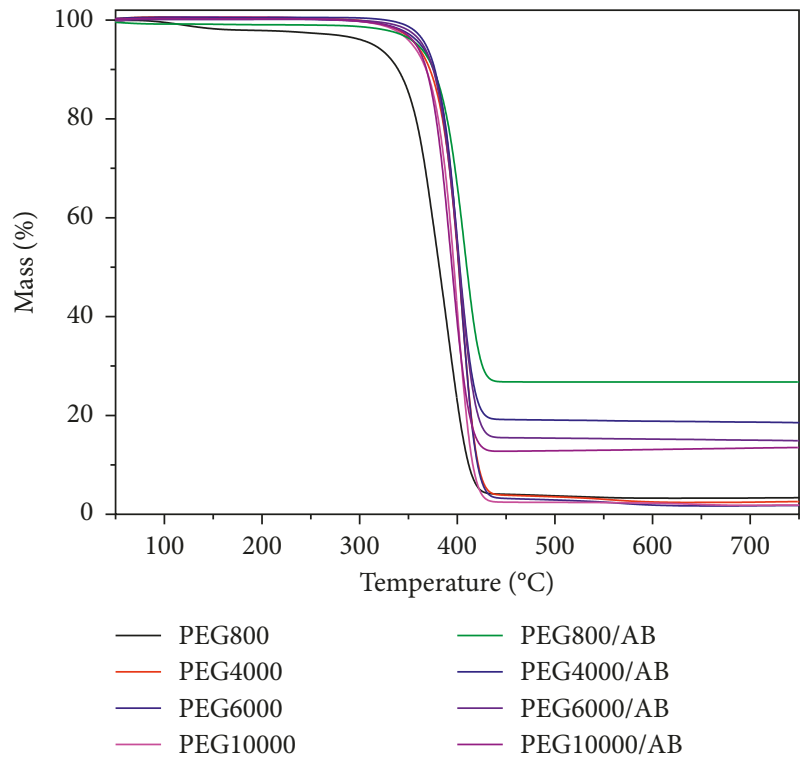

FIgure 4: TG plots of the PEGs and PEG/AB composites.

melt blending process, which demonstrates that the pristine PEG and the PEG/AB have the similar crystal structure and crystal cell type.

3.5. Thermal Conductivities of the PEG/AB Composites. The thermal conductivities of different mass ratios of $A B$ are presented in Figure 6. It can be seen that almost linear correlation exists between the thermal conductivity and content of the AB for each PEG system. A slow increase in thermal conductivity from $\sim 0.15 \mathrm{~W} / \mathrm{mK}$ to

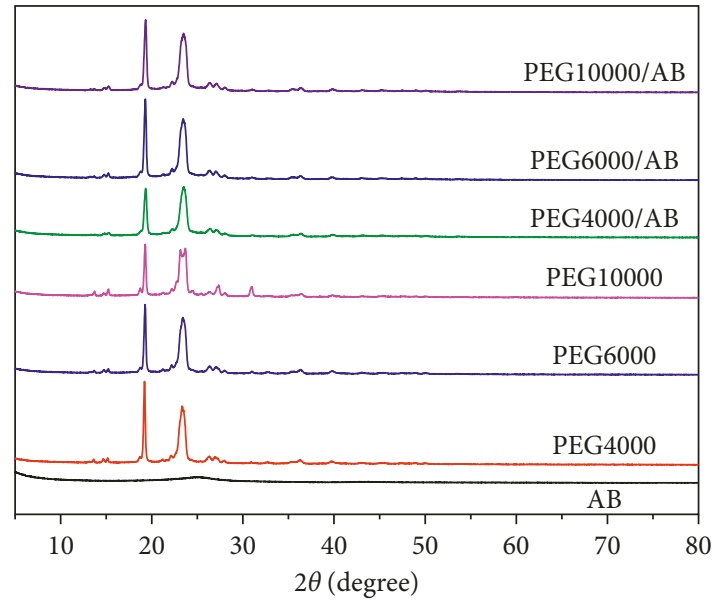

FIgUre 5: XRD spectra of the $\mathrm{AB}$, pristine PEGs, and PEG/AB composites.

$\sim 0.26 \mathrm{~W} / \mathrm{mK}$ with the increase of the mass ratio of the $\mathrm{AB}$ is observed due to the intrinsically low thermal conductivity of the $\mathrm{AB}$ induced by its amorphous structure. $A B$ is chosen as the supporting material to verify the effect of molecular weight of PEG on the shape stability so as to exclude the strong interactions between PEGs and supporting material, but actually it is not a promising thermally conductive filler for PCMs.

3.6. Thermal Performances of the PEG/AB Composites. The latent heat and phase change temperature of the samples are measured using DSC. Figure 7 shows the melting and freezing DSC curves of the pristine PEGs and form-stable PEG/AB 


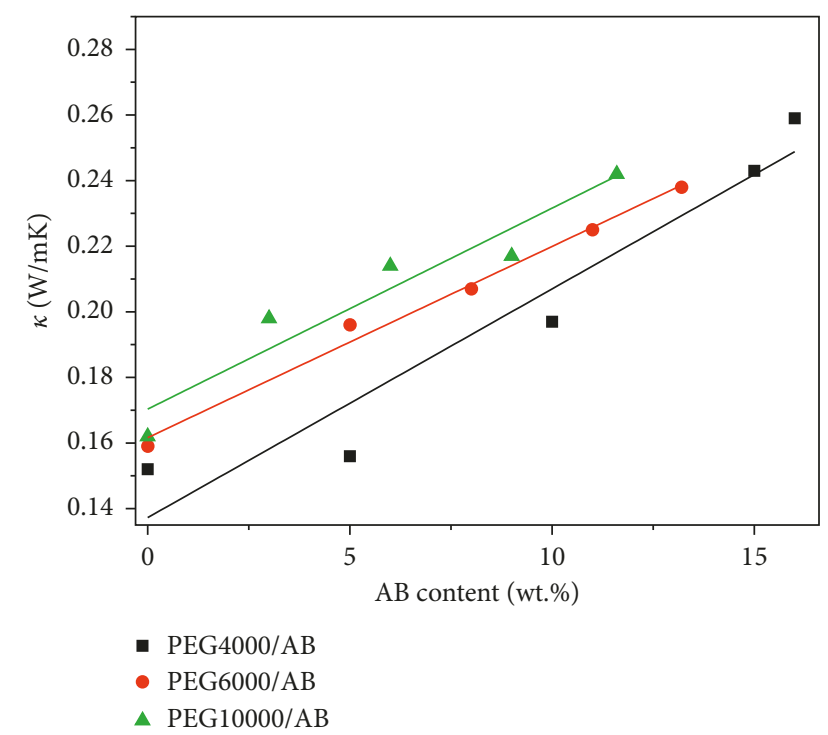

Figure 6: Thermal conductivities with various mass ratios of $\mathrm{AB}$.

composite PCMs. The detailed results of DSC thermal analysis of the peak melting/crystallization temperature $\left(T_{\mathrm{m}} / T_{\mathrm{c}}\right)$ ratio, the measured melting/crystallization enthalpy $\left(\Delta H_{\mathrm{m}} / \Delta H_{\mathrm{c}}\right)$, and the theoretical melting/crystallization enthalpy $\left(\Delta H_{\mathrm{m}}{ }^{*} / \Delta H_{\mathrm{c}}{ }^{*}\right)$ obtained are presented in Table 1 . The theoretical melting/crystallization enthalpies of the PEG/AB composites are calculated based on the impregnated contents of the PEG into the $\mathrm{AB}$ and the measured melting/crystallization enthalpies of pristine PEGs, assuming that the $\mathrm{AB}$ cannot contribute to the phase change latent heat.

The latent heat capacities of the composite PCMs containing the PEGs with different $M_{\mathrm{w}}$ are obviously lower than those of the pristine PEGs due to the incorporation of the $\mathrm{AB}$ that does not undergo phase change. Consequently, in most cases in this study, the melting enthalpies of the PEG/AB composites almost show agreement with the relevant mixture rules. The measured latent heats of the PEG/AB composites with varying PEG $M_{\mathrm{w}}$ from $\sim 4,000$ to $\sim 10,000$ are comparable with the theoretical latent heats. By adding a proper loading of the $\mathrm{AB}$, relatively high latent heats are retained, especially for the PEG/AB composites containing the PEGs with relatively large $M_{\mathrm{w}}$. The incorporation of the $\mathrm{AB}$ does not induce a significant change of the crystal structure of the PEG with relatively large $M_{\mathrm{w}}$. Notably, in the case of the PEG800/AB composite, the melting enthalpy is significantly lower by $55.7 \%$ than the theoretical value at the optimum loading ratio. This is partially expected given that some of the PCM weight (23.6\%) is replaced by the AB particles that are not subject to phase change. In addition, it is quite possible that other mechanisms also affect the phase change process of the PEG800 in the PEG800/AB composites. The presumed reason may be that some of the PEG chains are trapped inside the AB pores when $M_{\mathrm{w}}$ of the PEG is low (PEG800) due to the small equivalent sphere diameter of PEG800 compared to the most probable pore size of the $\mathrm{AB}$. The phase transition of the PEG from amorphous phase into stable crystal phase can be inhibited to a large extent due to the confined chain motion of the PEG induced by the strong adsorbability of the $A B$ pores, and thus the obviously reduced latent heat of phase change is detected by DSC. As a result, the phase change of the composite is prone to take place at lower temperature (Table 2). After $A B$ incorporation, the changes in crystallization temperature identify their tendency to supercool when cooling. However, the incorporation of the $\mathrm{AB}$ does not induce a significant supercooling of the PEGs with relatively large $M_{\mathrm{w}}$ because of decreased restriction from the $A B$ pores. By contrast, when the $M_{\mathrm{w}}$ of the PEG increased to $\sim 4,000$, most of the PEG chains are completely outside of the $A B$ pores. These chains, different from both the free PEG chains and the chains trapped into the $A B$ pores, are confined by the surface of the $A B$ particles. The longer PEG chains may offer more interacting site for the surface absorption of the PEG on the $A B$ particles. Furthermore, it should be noted that the optimum impregnation ratios of the PEG into the $A B$ for the PEG/AB composites increase with higher $M_{\mathrm{w}}$ of the PEG. These imply the existence of some other mechanisms leading to solid-solid phase change in the PEG/AB composite PCMs besides physical adsorptions. The phase change behavior of the PEG as a polymeric PCM is significantly different from small-molecule PCM, namely, much stronger intermolecular interactions than them. It thus explains in terms of the stronger intermolecular interactions, such as intermolecular friction and macromolecular entanglement, between the PEG4000 chains than those of the PEG800. Therefore, both the critical impregnated content of the PEG into the $\mathrm{AB}$ and enthalpy of the PEG4000/AB composite increase remarkably. When $M_{\mathrm{w}}$ increases to $\sim 6,000$, the confinement effect from intermolecular interactions is strengthened further with the increase of chain length, and thereby the declining amplitude in $T_{\mathrm{m}}$ of the $\mathrm{PEG} / \mathrm{AB}$ composite is reduced; the critical impregnated content of the PEG into the $\mathrm{AB}$ also shows an increasing trend. With the increase of $M_{\mathrm{w}}$ to 10,000 , the critical impregnated content of the PEG into the $A B$ increases further and thus the enthalpy of the PEG10,000/AB composite is improved as well. In other words, the phase change behaviors for the PEG/AB composites are not only attributed to the interactions between the $A B$ and PEG, but also to the intermolecular interactions of the PEG chains depending on the varying $M_{\mathrm{w}}$ of the PEG. The optimum absorption ratio of the PEG in the formstable PEG800/AB, PEG4,000/AB, PEG6,000/AB, and PEG10,000/AB composite PCMs reaches $0.76,0.84,0.87$, and 0.88 , respectively.

3.7. Shape-Stability Model of the PEG/AB Composites. A model is proposed to explain the shape stability of the PEG/AB composites, based on a physically crosslinked structure with temporary junctions (Figure 8). A part of PEG 


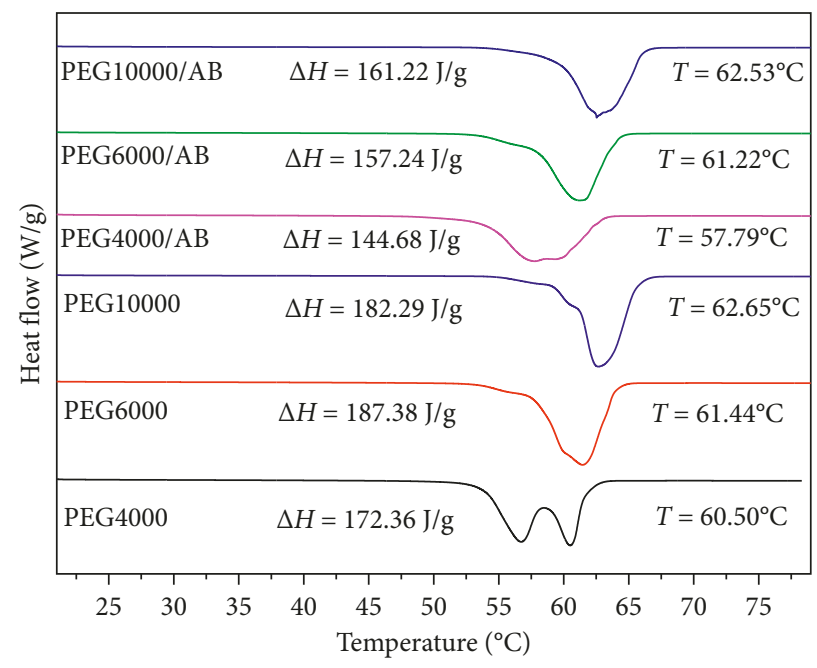

(a)

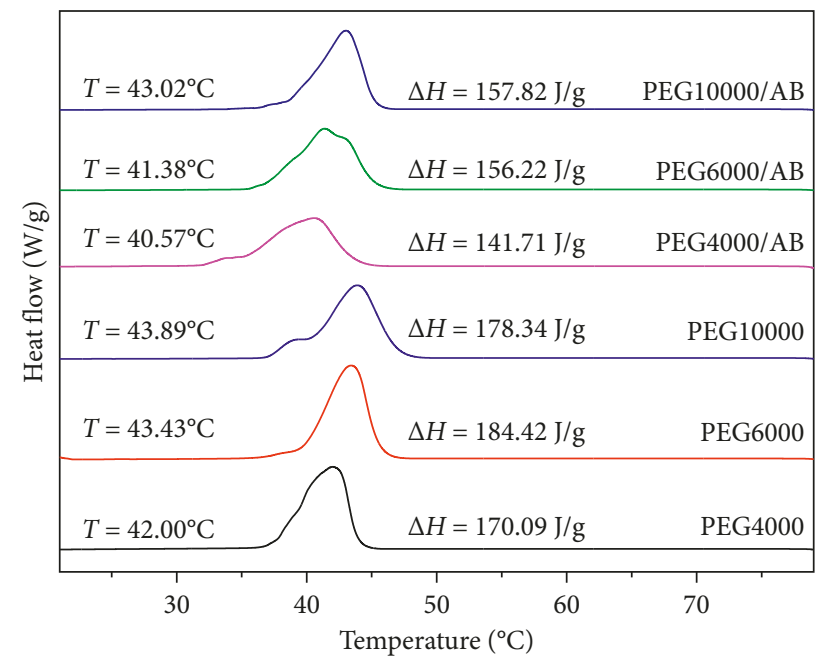

(b)

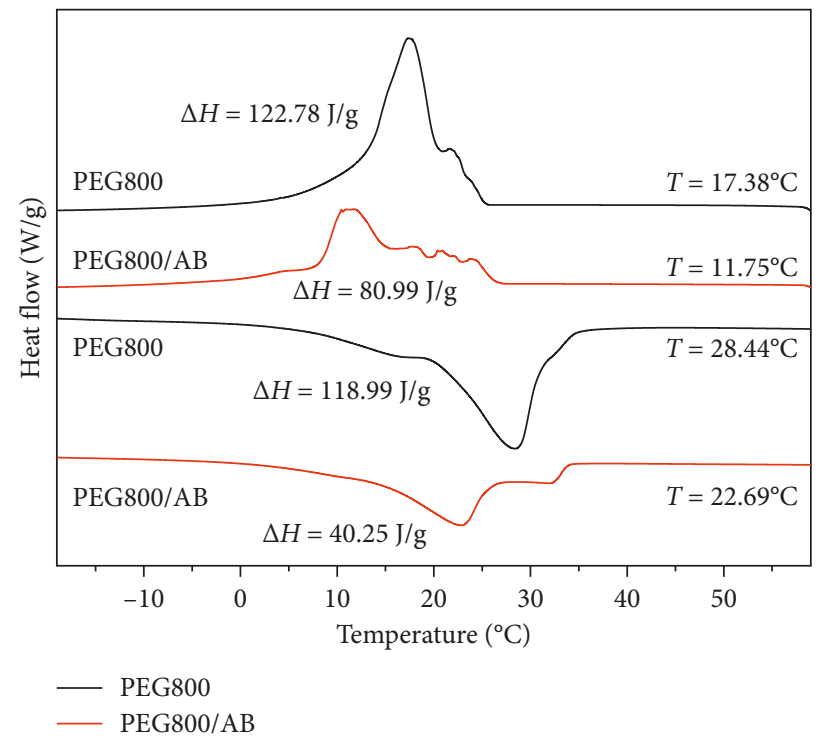

(c)

FIGURE 7: Cyclic DSC heating (a) and cooling (b) curves of pristine PEG4000, PEG6000, PEG10000, and their shape-stabilized PEG/AB composites. (c) DSC curves of PEG800 and PEG800/AB composites.

TABle 2: Thermal analysis of the PEG/AB composites and pure PEGs with varying molecular weights.

\begin{tabular}{lccccccc}
\hline Samples & Impregnation ratio (wt.\%) & $T_{\mathrm{m}}\left({ }^{\circ} \mathrm{C}\right)$ & $\Delta H_{\mathrm{m}}\left(\mathrm{J} \mathrm{g}^{-1}\right)$ & $\Delta H_{\mathrm{m}}{ }^{*}\left(\mathrm{~J} \cdot \mathrm{g}^{-1}\right)$ & $T_{\mathrm{c}}\left({ }^{\circ} \mathrm{C}\right)$ & $\Delta H_{\mathrm{c}}\left(\mathrm{J} \cdot \mathrm{g}^{-1}\right)$ & $\Delta H_{\mathrm{c}}{ }^{*}\left(\mathrm{~J} \cdot \mathrm{g}^{-1}\right)$ \\
\hline PEG800 & - & 28.44 & 118.99 & - & 17.38 & 122.78 \\
PEG4000 & - & 60.50 & 172.36 & - & 42.00 & 170.09 \\
PEG6000 & - & 61.44 & 187.38 & - & 43.43 & 184.42 & - \\
PEG10000 & - & 62.65 & 182.29 & - & 43.89 & 178.34 \\
PEG800/AB & 76.40 & 22.69 & 40.25 & 90.91 & 11.75 & 80.99 & - \\
PEG4000/AB & 83.90 & 57.79 & 144.68 & 144.68 & 40.57 & 141.71 \\
PEG6000/AB & 86.80 & 61.22 & 157.24 & 162.65 & 41.38 & 156.22 \\
PEG10000/AB & 88.40 & 62.53 & 161.22 & 161.14 & 43.02 & 157.82 & 160.08 \\
\hline
\end{tabular}

random coils with small equivalent radius and segments are trapped into the pores of the $\mathrm{AB}$ particles by physical interactions, such as capillary and surface tension forces (abbreviated as trapped PEG in Figure 8). In addition, some of the PEG chains that are closely adjacent to the $A B$ are adsorbed on the surface of the $\mathrm{AB}$ particles by hydrogen 

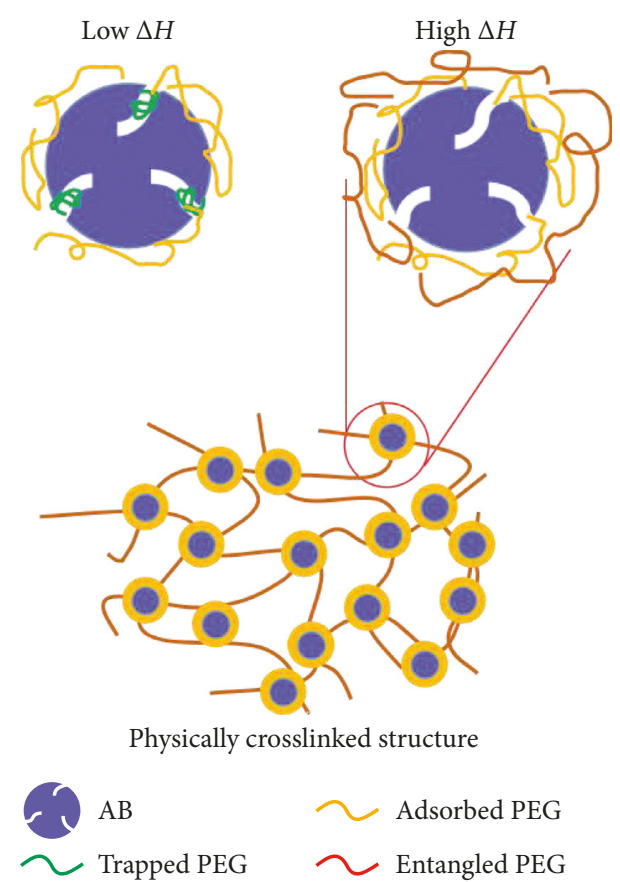

FIGURE 8: Schematic representation of shape stability.

bonding (abbreviated as adsorbed PEG in Figure 8); and some of the PEG chains with relatively large $M_{\mathrm{w}}$ are stabilized by the macromolecular entanglement and intermolecular friction (abbreviated as entangled PEG in Figure 8). The melting process of the PEG in the composite is confined by a physically crosslinked structure with temporary junctions above $T_{\mathrm{m}}$ of the PEG, which inhibits the phase change of form-stabilized solid phase into liquid phase. The structure becomes more stable with longer length of the PEG molecular chains, resulting in higher critical impregnated content of the PEG into the $\mathrm{AB}$. When the PEG content is increased further above the critical impregnated content, some of the PEG chains would be unbound above the phase change point of the composite, eliminating the shape-stabilized composites.

3.8. Structural Analysis of the PEG/AB Composites. Comparison between the $\mathrm{AB}$ and the $\mathrm{PEG} / \mathrm{AB}$ composites is made by SEM. Figure 9 shows the SEM images of the AB before PCM impregnation and the form-stable PEG/AB composites containing the critical impregnated contents of the PEGs. The morphology of the PEG800/AB composite is similar to that of the $\mathrm{AB}$ particles. The $\mathrm{AB}$ particles as the supporting material are covered with PEG layers, and the structure of $A B$ framework can be seen because the PEG layer is thin. A part of the PEG800 diffuses into the pores of the $\mathrm{AB}$ particles, which is consistent with the DSC results. As discussed before, the PEG molecules with relatively small $M_{\mathrm{w}}$ can be easily trapped by $\mathrm{AB}$, despite the fact that the $\mathrm{AB}$ can only provide limited adsorption capability due to the relatively low specific surface area and small pore size. In the case of the composite containing the PEG with relatively large $M_{\mathrm{w}}$, they appear to follow the different trend. Through SEM analysis, we confirm that most of the PEGs are not dispersed into the pores of the $\mathrm{AB}$. With an increase of $M_{\mathrm{w}}$ to $\sim 4,000$ (Figure 9(c)), the $\mathrm{AB}$ framework is not obvious since the PEG layer outside of the $\mathrm{AB}$ is thicker. When $M_{\mathrm{w}}$ increases to $\sim 6,000$ (Figure $9(\mathrm{~d})$ ) or $\sim 10,000$ (Figure $9(\mathrm{e})$ ), the $A B$ is covered with PEG completely and an irregular morphology can be found since the PEG layer is much thicker due to the longer molecular chain and higher mass fraction of the PEG. Based on the physically crosslinked structure model as discussed above, a part of the PEG segments and random coils with small equivalent radius are trapped into pores of the $\mathrm{AB}$ particles by capillary or surface tension forces. The hydrophilic character of the $\mathrm{AB}$ particles and cavities in them help us to improve the interfacial compatibility and wetting between the PEG and $A B$ particles; thus some of the PEG chains adjacent to the $\mathrm{AB}$ are adsorbed on $\mathrm{AB}$ particle surfaces by hydrogen bonding, capillary, or surface tension forces. Other PEG chains are anchored by intermolecular friction and entanglement because there are more chances of intermolecular interactions between the longer PEG chains compared to the PEG with relatively low $M_{\mathrm{w}}$. Physically crosslinked structure comprising the PEG chains and AB particles is formed through hydrogen bonding, capillary, surface tension forces, intermolecular friction, and macromolecular entanglement, which contributes to constrained chain motion and thus the solid-solid phase change behavior of the PEG/AB composites. Therefore, the optimum impregnation ratio of the $P E G$ into the $A B$ for the $P E G / A B$ composites without seepage of the melted PEG increases with the increasing $M_{\mathrm{w}}$ of the PEG.

\section{Conclusions}

The PEG/AB composite PCMs exhibited desirable properties in term of shape stability and large latent heats. The $\mathrm{AB}$ particles kept the composite form stable without inducing a large reduction in their phase change enthalpies. The fusion enthalpies of the form-stable PEG4,000/AB, PEG6,000/AB, and PEG10,000/AB composite PCMs reached 144.68, 157.24, and $161.22 \mathrm{~J} / \mathrm{g}$, respectively. Our results suggest that the phase change behaviors for the PEG/AB composites are attributed not only to the interactions between the $A B$ and PEGs but also to the intermolecular interactions of the PEG chains depending on the varying $M_{\mathrm{w}}$ of the PEGs. The shape stability of the PEG/AB composites were explained based on a physically crosslinked structure model. Physically crosslinked structure with temporary junctions was formed through hydrogen bonding, capillary, surface tension forces, intermolecular friction, and macromolecular entanglement. The structure became stabler with increasing length of the PEG molecular chains, resulting in the increase of the critical impregnated content of the $\mathrm{PEG}$ into the $\mathrm{AB}$. 


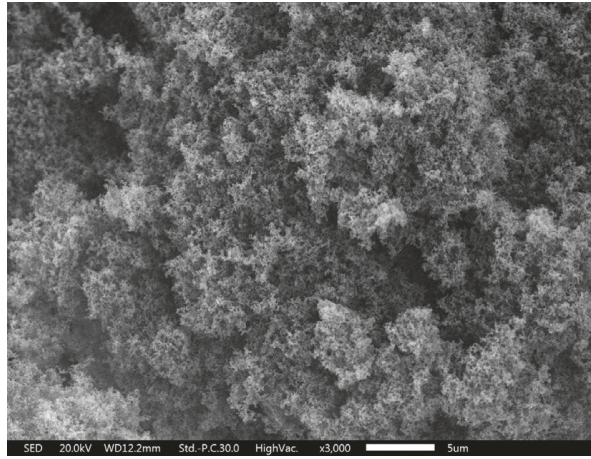

(a)

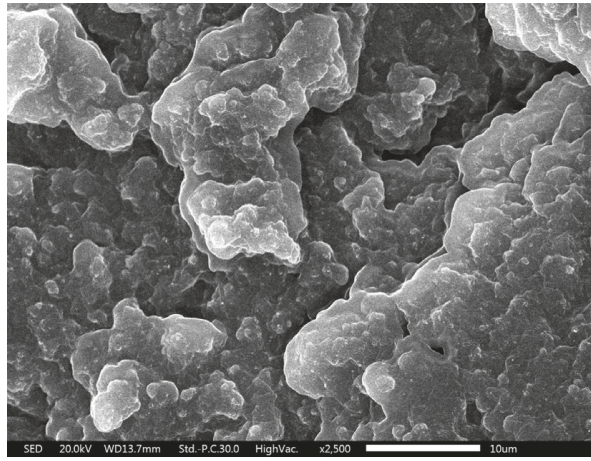

(c)

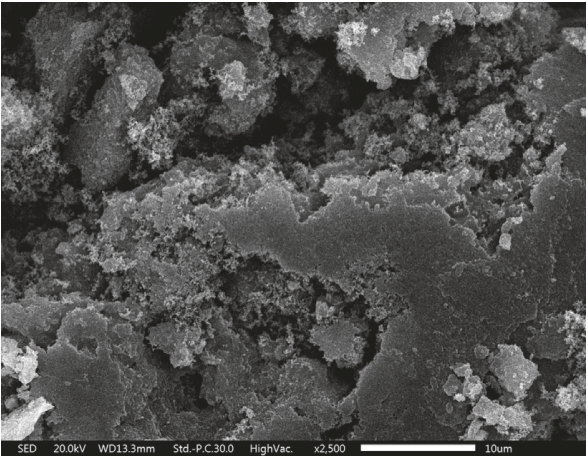

(b)

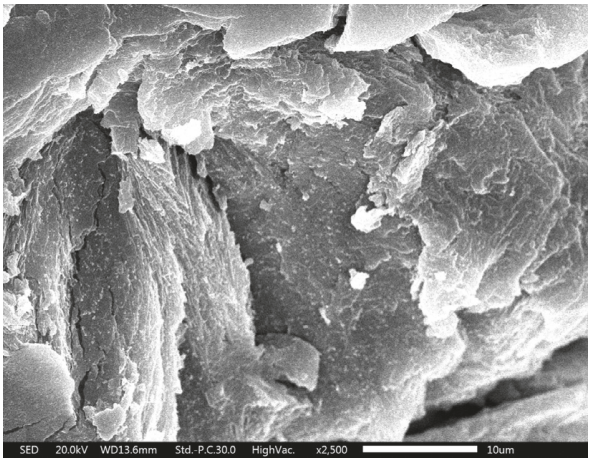

(d)

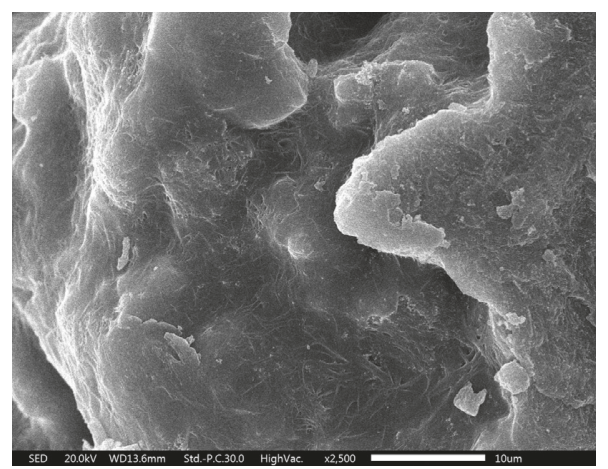

(e)

FIgure 9: SEM images of the shape-stabilized composite PCMs. (a) AB. (b) PEG800/AB. (c) PEG4000/AB. (d) PEG6000/AB. (e) PEG10000/AB.

\section{Data Availability}

The data used to support the findings of this study are available from the corresponding author upon request.

\section{Conflicts of Interest}

The authors declare that there are no conflicts of interest regarding the publication of this paper.

\section{Acknowledgments}

We gratefully acknowledge the support by the National Natural Science Foundation of China (No. 51503158).

\section{References}

[1] Q. Li, L. Chen, M. R. Gadinski et al., "Flexible hightemperature dielectric materials from polymer nanocomposites," Nature, vol. 523, no. 7562, pp. 576-579, 2015.

[2] B. Zalba, J. M. Marín, L. F. Cabeza, and H. Mehling, "Review on thermal energy storage with phase change: materials, heat transfer analysis and applications," Applied Thermal Engineering, vol. 23, no. 3, pp. 251-283, 2003.

[3] M. Mehrali, S. T. Latibari, M. Mehrali, H. Metselaar, and M. Silakhori, "Shape-stabilized phase change materials with high thermal conductivity based on paraffin/graphene oxide composite," Energy Conversion and Management, vol. 67, pp. 275-282, 2013. 
[4] A. Abhat, "Low temperature latent heat thermal energy storage: heat storage materials," Solar Energy, vol. 30, no. 4, pp. 313-332, 1983.

[5] S. D. Sharma and K. Sagara, "Latent heat storage materials and systems: a review," International Journal of Green Energy, vol. 2, no. 1, pp. 1-56, 2005.

[6] Y. Tian and C. Y. Zhao, "A Review of solar collectors and thermal energy storage in solar thermal applications," Applied Energy, vol. 104, pp. 538-553, 2013.

[7] A. Váz Sá, R. Almeida, H. Sousa, and J. Delgado, "Numerical analysis of the energy improvement of plastering mortars with phase change materials," Advances in Materials Science and Engineering, vol. 2014, Article ID 245473, 13 pages, 2014.

[8] C. Alkan, A. Sari, and O. Uzun, "Poly (ethylene glycol)/acrylic polymer blends for latent heat thermal energy storage," AIChE Journal, vol. 52, no. 9, pp. 3310-3314, 2006.

[9] K. Pielichowski and K. Flejtuch, "Differential scanning calorimetric studies on poly(ethylene glycol) with different molecular weights for thermal energy storage materials," Polymers for Advanced Technologies, vol. 13, no. 10-12, pp. 690-696, 2002.

[10] S. Han, C. Kim, and D. Kwon, "Thermal degradation of poly (ethylene glycol)," Polymer Degradation and Stability, vol. 47, no. 2, pp. 203-208, 1995.

[11] H. Li, M. Jiang, Q. Li et al., "Aqueous preparation of polyethylene glycol/sulfonated graphene phase change composite with enhanced thermal performance," Energy Conversion and Management, vol. 75, pp. 482-487, 2013.

[12] X. Jiao, D. Zhao, Y. Zhang et al., "Microencapsulated dimethyl terephthalate phase change material for heat transfer fluid performance enhancement," Colloid and Polymer Science, vol. 294, no. 4, pp. 639-646, 2016.

[13] H. Li, M. Jiang, Q. Li et al., "Facile preparation and thermal performances of hexadecanol/crosslinked polystyrene core/ shell nanocapsules as phase change material," Polymer Composites, vol. 35, no. 11, pp. 2154-2158, 2014.

[14] A. Sarı, C. Alkan, and A. Biçer, "Synthesis and thermal properties of polystyrene-graft-PEG copolymers as new kinds of solid-solid phase change materials for thermal energy storage," Materials Chemistry and Physics, vol. 133, no. 1, pp. 87-94, 2012.

[15] R. Cao, H. Liu, S. Chen, D. Pei, J. Miao, and X. Zhang, "Fabrication and properties of graphene oxide-grafted-poly (hexadecyl acrylate) as a solid-solid phase change material," Composites Science and Technology, vol. 149, pp. 262-268, 2017.

[16] N. Sarier, E. Onder, S. Ozay, and Y. Ozkilic, "Preparation of phase change material-montmorillonite composites suitable for thermal energy storage," Thermochimica Acta, vol. 524, no. 1-2, pp. 39-46, 2011.

[17] X. Fu, Z. Liu, B. Wu, J. Wang, and J. Lei, "Preparation and thermal properties of stearic acid/diatomite composites as form-stable phase change materials for thermal energy storage via direct impregnation method," Journal of Thermal Analysis and Calorimetry, vol. 123, no. 2, pp. 1173-1181, 2016.

[18] D. Mei, B. Zhang, R. Liu, Y. Zhang, and J. Liu, "Preparation of capric acid/halloysite nanotube composite as form-stable phase change material for thermal energy storage," Solar Energy Materials and Solar Cells, vol. 95, no. 10, pp. 27722777, 2011.

[19] L. Feng, J. Zheng, H. Yang, Y. Guo, W. Li, and X. Li, "Preparation and characterization of polyethylene glycol/ active carbon composites as shape-stabilized phase change materials," Solar Energy Materials and Solar Cells, vol. 95, no. 2, pp. 644-650, 2011.

[20] Y. Wang, T. D. Xia, H. Zheng, and H. X. Feng, "Stearic acid/ silica fume composite as form-stable phase change material for thermal energy storage," Energy and Buildings, vol. 43, no. 9, pp. 2365-2370, 2011.

[21] Z. Zhang, N. Zhang, J. Peng, X. Fang, X. Gao, and Y. Fang, "Preparation and thermal energy storage properties of paraffin/expanded graphite composite phase change material," Applied Energy, vol. 91, no. 1, pp. 426-431, 2012.

[22] J. Yang, G. Qi, Y. Liu et al., "Hybrid graphene aerogels/phase change material composites: thermal conductivity, shapestabilization and light-to-thermal energy storage," Carbon, vol. 100, pp. 693-702, 2016.

[23] K. Yuan, Y. Zhou, W. Sun, X. Fang, and Z. Zhang, "A polymer-coated calcium chloride hexahydrate/expanded graphite composite phase change material with enhanced thermal reliability and good applicability," Composites Science and Technology, vol. 156, pp. 78-86, 2018.

[24] D. H. Atha and K. C. Ingham, "Mechanism of precipitation of proteins by polyethylene glycols. Analysis in terms of excluded volume," Journal of Biological Chemistry, vol. 256, no. 23, pp. $12108-12117,1981$. 


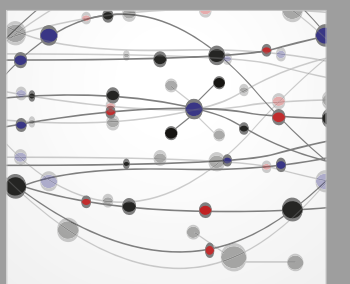

The Scientific World Journal
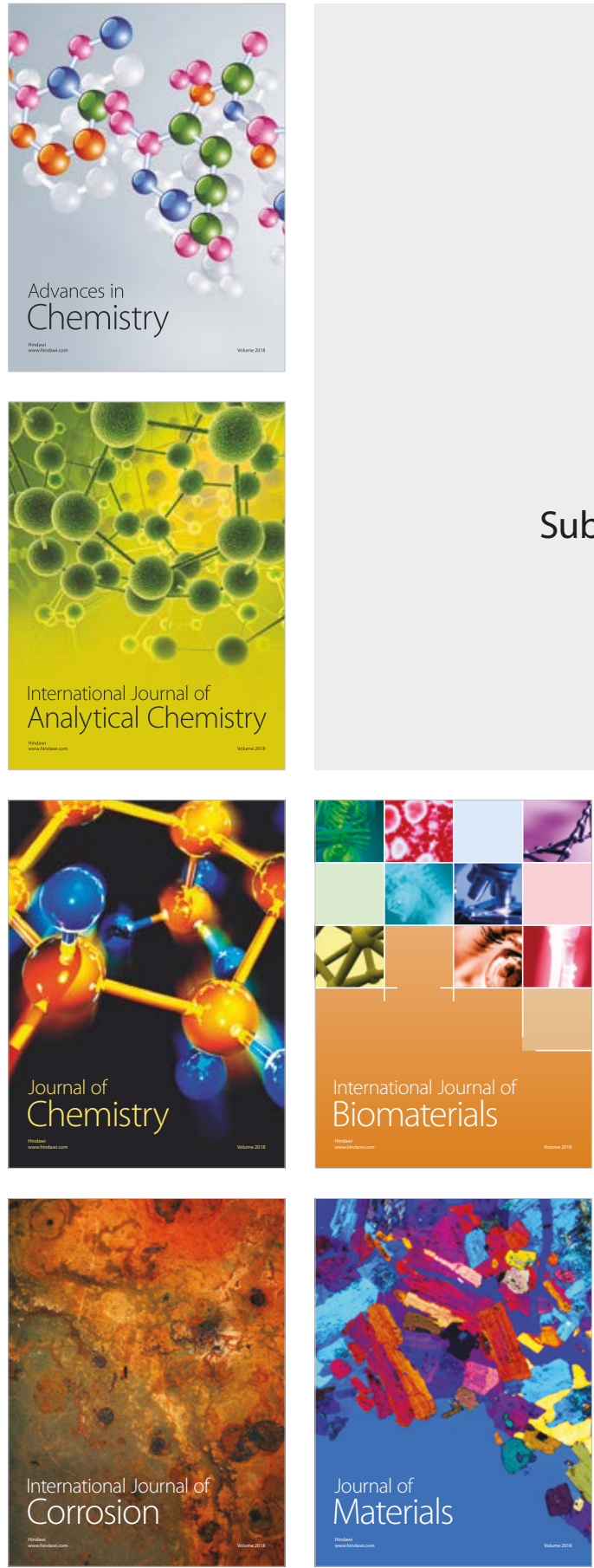

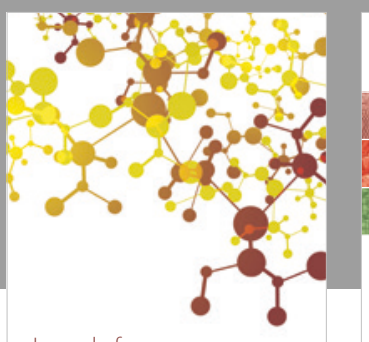

Journal of

Applied Chemistry
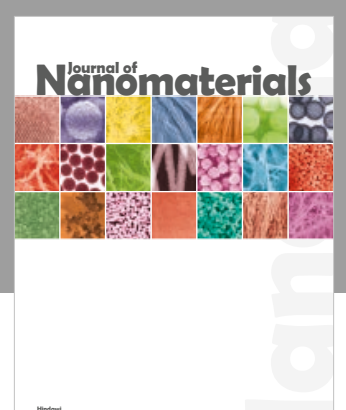

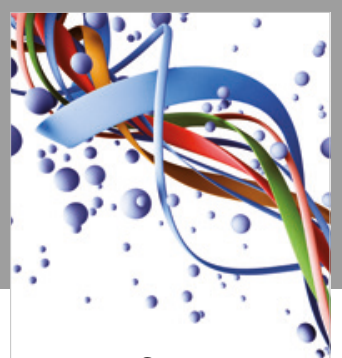

Scientifica

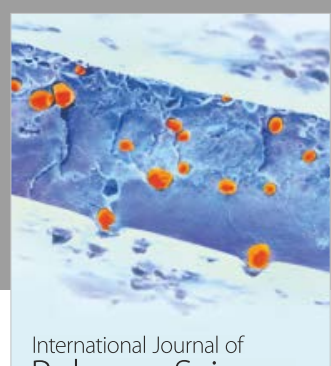

Polymer Science

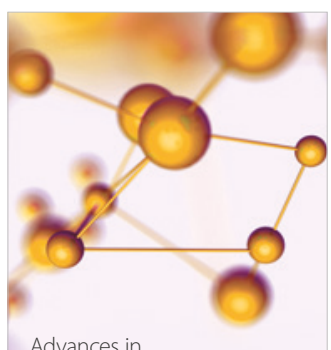

Physical Chemistry
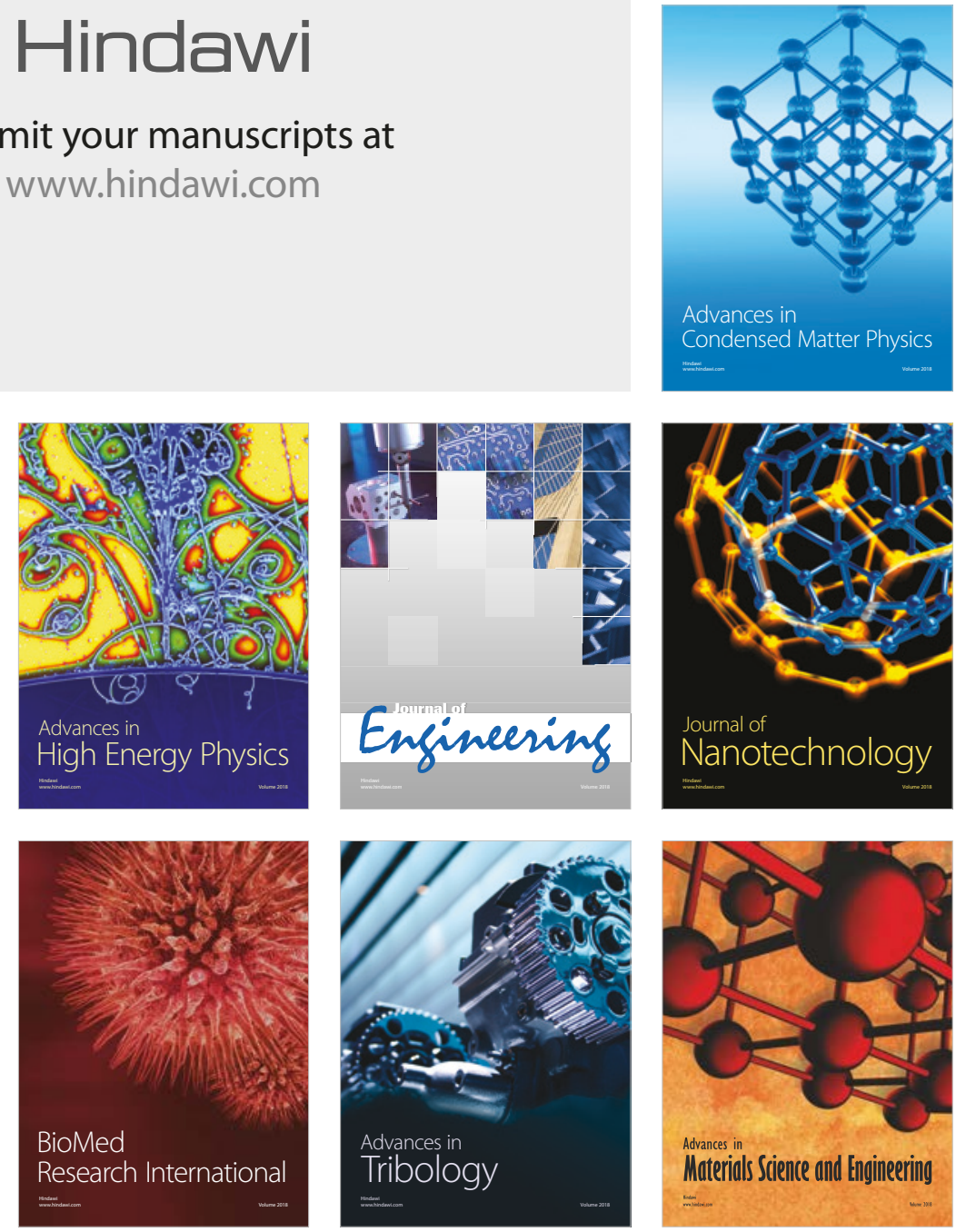